\title{
Use of Vacuum-Assisted Closure in the Management of Colostomy
}

\author{
Simone Zanella and Alberto Di Leo
}

\begin{abstract}
Background: This article is the first report of the simultaneous use of vacuum-assisted closure (VAC) therapy and an oral tube applied as a life-saving treatment for colostomy dehiscence to avoid fecal contamination.

Case Presentation: A 75-year-old female was admitted to our surgical unit in 2012 for knee trauma caused by an accidental fall at home. Surgery for lysis of adhesions, left colectomy, colorectal anastomosis, and protective ileostomy was performed initially for sigmoidovaginal fistula. Vacuum-assisted closure therapy (applying continuous negative abdominal pressure) was used after median wound dehiscence and a parastomal abscess that caused the necrosis of the skin and subcutaneous tissue surrounding the colostomy.

Conclusion: The application of a negative pressure is a novel approach to colostomy dehiscence and complicated abdominal wound management and can lead to complete abdominal wound closure and finally to apply a standard plate for colostomy bag.
\end{abstract}

Keywords: colostomy; parastomal abscess; wound infection

$\mathbf{V}$ ACUUM-ASSISTED CLOSURE (VAC) is a system first reported in 1997 [1,2] in plastic surgery. The benefit of VAC therapy has been established for diabetic foot wounds, chronic leg ulcers, skin grafts for burns, and post-operative wound infections after trauma or vascular surgery [3]. Applied controlled negative pressure decreases bacterial colonization, tissue edema, wound tension, increases blood flow, and promotes wound granulation [4]. This approach is now also being used the management of complex abdominal wounds [5]. Vacuum-assisted closure has recently been used in gastrointestinal surgery field and for open abdominal wounds [5] with a shortened time for reconstruction and more recently to treat partial stoma dehiscence [6].

Anastomotic dehiscence is a complication in colorectal surgery. A recent systematic review showed that the overall rate of dehiscence was $8.6 \%$, the rate of dehiscence requiring re-operation was $5.4 \%$, the post-operative mortality was $0.4 \%$, and the overall post-operative mortality was $1.3 \%$ [7]. We report a case of complete stoma dehiscence for abscess after colorectal surgery that was treated successfully using VAC therapy.

\section{Case Presentation}

A 75-year-old female was admitted to our surgical unit in 2012 for knee trauma resulting from an accidental fall at home. Medical history included bilateral hip, vascular encephalopathy, osteoporosis, polyarthrosis in steroid therapy, steroid diabetes mellitus, and remote bilateral hysteroadnexectomy complicated by the development of an incisional hernia, and removal of pituitary macroadenoma three years prior. The patient reported a serious deterioration of the general condition with a 9-kg weight loss over approximately two months (body mass index [BMI $32 \mathrm{~kg} / \mathrm{m}^{2}$ at admission), the appearance of multiple ulcerations of the lower limbs and in the sacral region, and the appearance of abundant dark and odorous vaginal discharge three to four months prior. Tests performed during recovery, specifically pelvic examination, colonoscopy, cystography, and colpography, led to the diagnosis of left colon diverticular disease complicated by sigmoidovaginal fistula. The patient underwent surgical operation of lysis of adhesions, left colectomy, colorectal anastomosis, and protective ileostomy on the right side.

Department of Surgery, S. Maria del Carmine Hospital, Rovereto, Italy.

(c) Simone Zanella and Alberto Di Leo 2016; Published by Mary Ann Liebert, Inc. This Open Access article is distributed under the terms of the Creative Commons License (http://creativecommons.org/licenses/by/4.0), which permits unrestricted use, distribution, and reproduction in any medium, provided the original work is properly credited. 


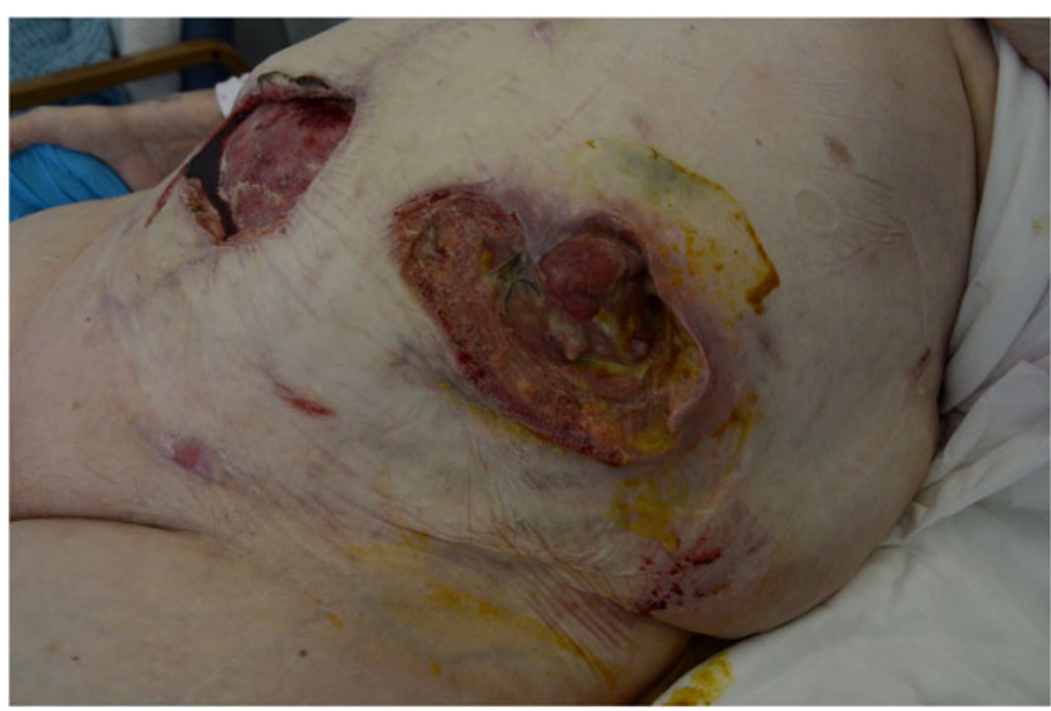

FIG. 1. Wound dehiscence median and a parastomal abscess that caused the necrosis of the skin and subcutaneous tissue surrounding the colostomy.
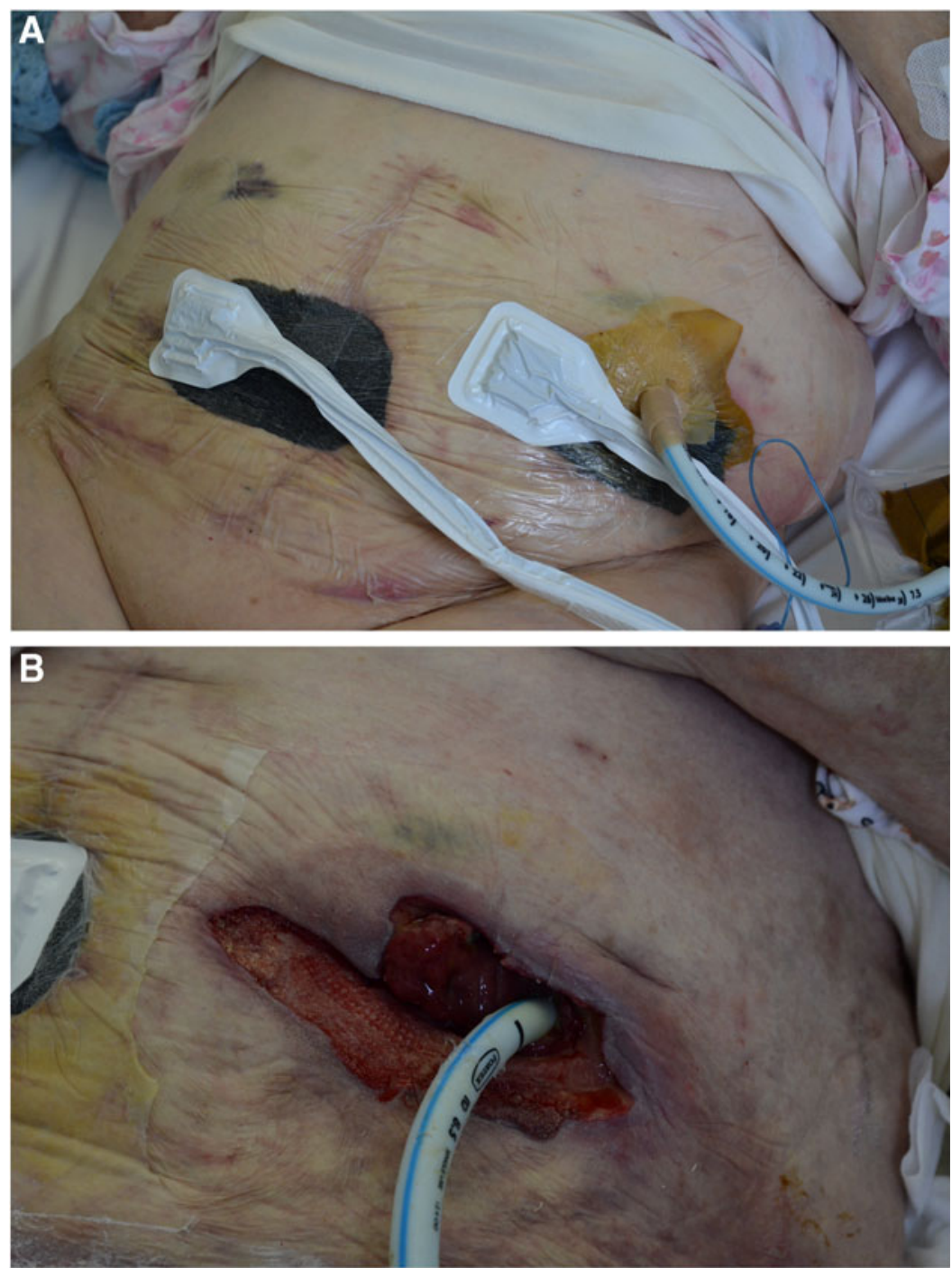

FIG. 2. (A) and (B) Cuffed oral tube in permitted to maintain cleaning the wound and to avoid fecal contamination. 


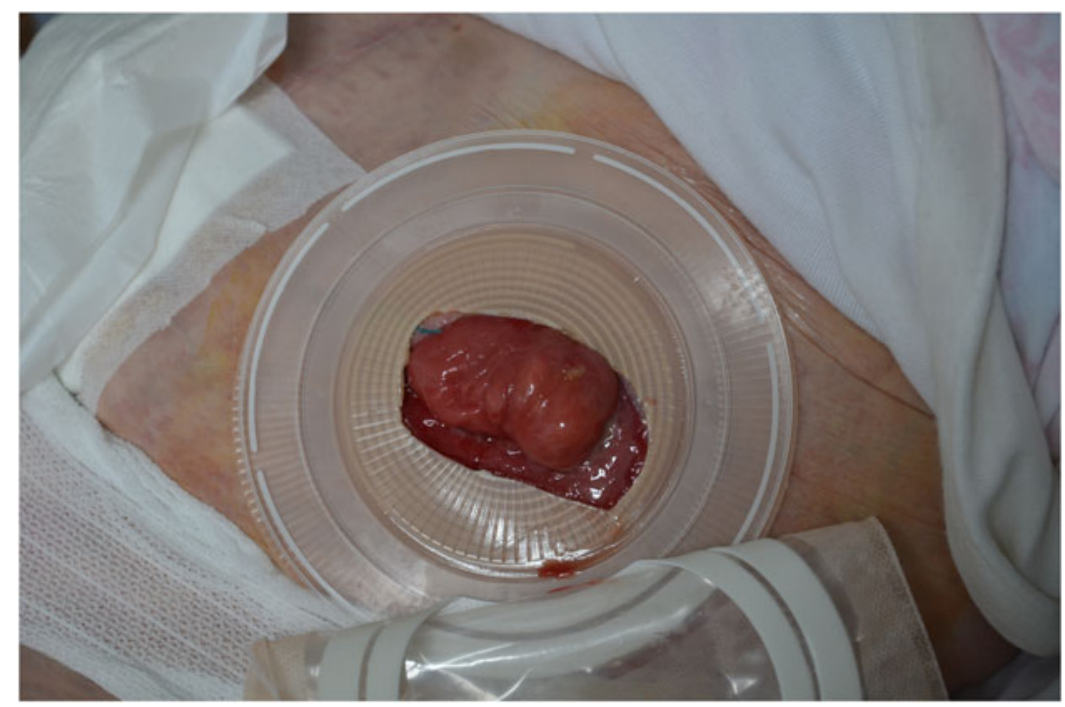

FIG. 3. After three months of vacuum-assisted closure (VAC) treatment application of a standard plate for colostomy bag.

Seven days post-operatively the patient developed dehiscence of inferior part of ileostomy and dehiscence of colorectal anastomosis with diffuse peritonitis.

We decided to perform emergency re-laparotomy, closing protective ileostomy, and creating a colostomy on the left side. The subsequent post-operative period was complicated by median incision dehiscence and a parastomal abscess that caused the necrosis of the skin and subcutaneous tissue surrounding the colostomy (Fig. 1).

After trying unsuccessfully to achieve healing of the median wound and approximation with sutures of colostomy margins skin, after four weeks it was decided to place the VAC on both areas, adjusting it to a pressure varying from $-80 \mathrm{~mm} \mathrm{Hg}$ to $-120 \mathrm{~mm} \mathrm{Hg}$. The simultaneous placement of a cuffed oral tube in left colostomy permitted to maintain cleaning the wound (Fig. 2A and 2B) and to avoid fecal contamination. After three months of VAC treatment, it was possible to do a skin graft over the median wound and apply a standard plate for colostomy bag (Fig. 3). The patient was discharged after two weeks without complications.

\section{Discussion and Literature Review}

Surgical site infection (SSI) has a prevalence of $2 \%-5 \%$ and increases the cost of surgical patient care by approximately $20 \%$. Diabetes mellitus and obesity are two of the main risk factors of such infections. Another important factor that may compromise the SSI healing is impaired tissue perfusion.

Clinical application of negative pressure was first used in Chinese medicine techniques with acupuncture for its ability to cause hyperemia, resulting in increased local circulation through vasodilation. Historically, different systems have been developed to use the negative pressure in the treatment of wounds. Vacuum-assisted closure is a system first reported in 1997 [1,2] in plastic surgery. The polyurethane foam used with a regular pattern of large holes $(400-600 \mathrm{mcm})$ allows an even distribution of pressure over the entire surface of the wound.

Vacuum-assisted closure has been used recently for open abdominal wound dehiscence [5] and for partial stoma dehiscence [6]. Crick et al. [6] reported a case of wound dehiscence of mid-line laparotomy and the end ileostomy in a 41-year-old female operated on for fecal peritonitis. In our case, stoma dehiscence was not of ileostomy, but colostomy and both patients were obese. To avoid wound contamination we used an oral tube to drain colostomy, first case reported in literature associated with VAC therapy, also because of different material was drained from colostomy despite ileostomy. We obtained a complete resolution of the problem in three months unlike Crick et al. [6] who used a higher negative pressure over six months.

Risk factors of dehiscence are advanced age, malnutrition, pulmonary disease, renal failure, obesity, diabetes mellitus, steroid therapy, radiotherapy, and chemotherapy [8]. Many of these were present in the case considered above.

Post-operative complications after enterostomy are common with a prevalence rate of $72 \%$. The above case refers to colostomy dehiscence caused by the evolution of a parastomal abscess. It was associated with a high risk of infection of the soft tissues of the abdominal wall and resulted in the inability to place a standard plate for a normal colostomy management. Vacuum-assisted closure placed over eight weeks obtained the optimal condition to perform a skin graft on the wall and to apply a standard plate for colostomy.

\section{Conclusion}

In conclusion, we found the VAC therapy a life-saving method for the management of dehiscence colostomy. With complete resolution of the problem it can lead to complete abdominal wound closure and finally to apply a standard plate for colostomy bag.

\section{Author Disclosure Statement}

This study was not supported by any grants.

\section{References}

1. Argenta LC, Morykwas MJ. Vacuum-assisted closure: A new method for wound control and treatment: Clinical experience. Ann Plast Surg 1997;38:563-577.

2. Morykwas MJ, Argenta LC, Shelton-Brown EI, McGuirt W. Vacuum-assisted closure: A new method for wound control 
and treatment: Animal studies and basic foundation. Ann Plast Surg 1997;38:553-562.

3. Saxena V, Hwang CW, Huang S, et al. Vacuum-assisted closure: Microdeformations of wounds and cell proliferation. Plast Reconstr Surg 2004;114:1086-1096.

4. Fujino S, Miyoshi N, Ohue M, et al. Use of vacuum-assisted closure in management of open abdominal wound with multiple enterocutaneous fistulae during chemotherapy: A case report. Int J Surg Case Rep 2015;17:112-116.

5. Vuerstaek JD, Vainas T, Wuite J, et al. State-of-the-art treatment of chronic leg ulcers: A randomized controlled trial comparing vacuum-assisted closure (V.A.C.) with modern wound dressings. J Vasc Surg 2006;44:1029-1038.

6. Crick S, Roy A, Macklin CP. Stoma dehiscence treated successfully with VAC dressing system. Tech Coloproctol 2009;13:181.

7. Cong ZJ, Hu LH, Xing JJ, et al. Incidence and mortality of anastomotic dehiscence requiring reoperation after rectal carcinoma resection. Int Surg 2014;99:112-119.

8. Rojas-Machado SA, Romero-Simó M, Arroyo A, et al. Prediction of anastomotic leak in colorectal cancer surgery based on a new prognostic index PROCOLE (prognostic colorectal leakage) developed from the meta-analysis of observational studies of risk factors. Int J Colorectal Dis 2016;31:197-210.
Address correspondence to:

Dr. Simone Zanella

General Surgery

S. Maria del Carmine Hospital Corso Verona 4 38068 Rovereto

Italy

E-mail: simonezanella@apss.tn.it

$\begin{aligned} & \text { Abbreviations Used } \\ \mathrm{BMI} & =\text { body mass index } \\ \mathrm{SSI} & =\text { surgical site infection } \\ \mathrm{VAC} & =\text { vacuum-assisted closure }\end{aligned}$

Cite this article as: Zanella S, Di Leo A (2016) Use of vacuum-assisted closure in the management of colostomy: A case report. Surgical Infections Case Reports 1:1, 165-168, DOI: 10.1089/crsi.2016.0043 